\title{
La visión de los archivos en el Antiguo Régimen hispano a través de las memorias y relatos de viajeros: análisis de su representación y sistematización metodológica
}

\author{
Agustín Vivas Moreno \\ Carmen Solano Macías \\ Aitana Martos García \\ Universidad de Extremadura (España) \\ Ana Isabel Aznar Castro \\ María Rodríguez Lairado
}

\section{Resumen}

Informe metodológico de la historia de los archivos en el Antiguo Régimen basado en una de sus fuentes históricas: las memorias y relatos de viajeros. Se pretende realizar una sistematización metodológica para la consecución de una historia de los archivos a través de su representación en las fuentes indirectas y analizar la imagen archivística durante el Antiguo Régimen en España a partir de una de las fuentes de mayor interés: las memorias y los relatos de viajeros. La metodología utilizada ha sido seleccionar de forma empírica ciertos textos extraídos de memorias y relatos de viajeros, que tras el análisis reflejan representaciones y visiones diversas de archivos; y también de forma teórica y cualitativa, pretendiendo incardinar nuestro estudio en la historia social de lo cultural (R. Chartier), y en concreto de la cultura escrita; y además analizando los textos seleccionados en relación con el contexto histórico e institucional. Tras el trabajo, se han obtenido los siguientes resultados: las referencias extraídas reflejan opiniones, descripciones, valoraciones y juicios críticos de diverso encaje, y son representativos de los diferentes usos y funciones de la cultura escrita en el Antiguo Régimen hispano. Además, veremos ciertos factores que articulan la configuración de los archivos y la archivística. Por tanto, podemos concluir, por un lado, en la utilidad de las memorias y relatos de viajeros, y, por extensión, de las fuentes históricas y literarias, para el estudio de la visión de los archivos y su representación en el Antiguo Régimen hispano; y por otro, en la consideración de dichas herramientas para la confección de una historia de la archivística de larga duración, esto es, la historia cultural de la archivística. 
Palabras clave: Historia de la archivística. Historia cultural. Fuentes indirectas. Memorias de viajeros. Relatos de viajeros.

\section{Abstract}

A methodological report on the history of archives in the Ancien Regime in Spain is offered, based on one of its historical sources: travellers' life stories and tales. Several texts were extracted from traveller life stories and tales, based on previous knowledge of the sources, which were analysed in relation to their historical and institutional context. Such extracts capture varied opinions, descriptions, evaluations, and value judgements, and represent different customs and functions around the use of the Written Culture, archives and Archival Science in the Hispanic Ancien Regime. The utility of travellers' life stories and tales, and in general, of historical and literary sources, for the study of the vision of archives and their representation in the Hispanic Ancien Regime, and thereafter for a History of Archives and Archival Science, is established.

Palabras clave: History of Archival Science. Cultural History. Indirect information sources. Travellers' Lifestories. Travellers' Tales.

\section{Introducción}

El análisis de los archivos durante el Antiguo Régimen hispano es ciertamente complejo, de manera que los objetivos que nos proponemos deben quedar previamente ajustados. En cualquier caso, pretendemos incardinar el presente escrito en las corrientes de investigación sobre historia de los archivos y de la archivística, y, de forma concreta, en una historia de "ritmos lentos" que podríamos denominar historia cultural de la archivística de metodología sistémica, que aspira a construir un modelo o cuadro teórico adaptado al análisis de los diferentes sistemas socioculturales, entendidos precisamente como conjuntos de elementos interrelacionados estructuralmente.

Así pues, lo que ahora nos proponemos es doble: por un lado, perfilar las múltiples y dificultosas fuentes que, desde diferentes perspectivas, son de utilidad - unas de forma especifica y otras de modo más indirecto - para el estudio de la historia de los archivos y de la archivística durante los siglos XVI-XVIII hispanos; y por otro, centrarnos en una de las fuentes que resultan ser de enorme importancia para variados asuntos de marcado carácter histórico, y de aprovechamiento, en consecuencia, para el análisis del pasado de los archivos: las memorias y relatos de viajeros.

Estructuraremos, pues, nuestro escrito en tres apartados. En el primero de ellos, intentaremos descifrar de forma muy sucinta cómo las memorias y relatos de viajeros son una de las múltiples fuentes de utilidad para la configuración del 
necesario análisis pluridimensional de la historia de los archivos y de la archivística. En el segundo, proporcionaremos una explicación esquemática de la fuente en sí, esto es, daremos algunas indicaciones acerca de su tipología, avanzaremos sobre sus múltiples formas y finalidades, examinaremos sus características peculiares como fuente, indagaremos dónde radica su interés histórico y dónde su problemática y dificultades de uso, y sondearemos cuáles son sus elementos básicos y constitutivos o cuál su forma de presentación. Y en el tercero, nos centraremos en la visión que constatamos que los viajeros - durante el Antiguo Régimen hispano - tienen de los archivos y su contexto. Explicaremos cuál ha sido la metodología seguida y cuáles las ideas vertebradoras que caracterizan su visión. Abordemos, pues, el primer punto.

\section{Aproximación a las fuentes para el estudio de la historia de la archivística}

Debemos comenzar afirmando que la historia de los archivos y de la archivística es una de las líneas de investigación o categoría temática que la mayoría de las clasificaciones convencionales sobre temas de investigación en archivística distinguen (1). En este sentido, es un hecho constatable cómo se ha producido un incremento de la producción para este tema durante los años noventa, lo que indica un afán por establecer el nacimiento de la archivística como saber científico.

Sin embargo, la historiografía presenta aún ciertas carencias, que desde nuestro punto de vista pueden quedar esquematizadas de la siguiente forma. En primer lugar, la ausencia de obras interdisciplinares y estudios sintéticos, debida en gran medida a la necesidad de equipos interdisciplinares con investigadores altamente especializados para cada una de las etapas históricas. En segundo lugar, la escasez de obras que relacionen la historia de la archivística con su contexto administrativo e histórico. Son pocas las que existen, y, en la mayoría de los casos, se trata de investigaciones incluidas en obras genéricas que atienden fundamentalmente a los aspectos históricos y diplomáticos, prescindiendo en gran medida de los agentes intervinientes en los procesos administrativos. En consecuencia, serían deseables investigaciones de carácter histórico que analizaran la interdependencia entre los procesos administrativos y los archivísticos. En tercer lugar, constatamos una falta de repertorios de fuentes y ediciones de texto. Sería deseable conocer mayor número de fuentes con las que poder elaborar una historia de la archivística y editar aquellos textos de interés que muestren más claramente su evolución (2). Y por último, carecemos de obras de carácter metodológico para la multiplicidad de perspectivas que se ofrecen y determinados estudios monográficos. Y si para el primer caso el panorama empieza a ser esperanzador a través de las vinculaciones con los historiadores de la cultura escrita, de la literatura o de las instituciones, para el segundo caso aún carecemos de estudios que analicen, por

Scire. $11: 1$ (en.-jun. 2005) 47-64. ISSN 1135-3761. 
ejemplo, asuntos vinculados a la documentación de la época moderna, aspectos históricos de instituciones de relieve, análisis de procedencias de determinados fondos documentales o cuestiones de carácter organizativo para los archivos del Antiguo Régimen.

Evidentemente, dichas carencias se han visto impulsadas por dificultades de carácter intrínseco a la materia en cuestión, que son imponderables que, en ocasiones, resultan insoslayables. Dichas dificultades son: (a) la amplitud y complejidad temática de la disciplina motivada por la extraordinaria interdependencia con otras disciplinas; (b) la consiguiente dispersión bibliográfica en multitud de colecciones y revistas de diferentes materias, y con resultados desiguales y multiformes; y (c) la inexistencia de una coordinación institucional para el tema que guíe la multiplicidad fraccionada de abundantes obras.

Por otro lado, acercarse al estudio de las fuentes para la historia de los archivos y de la archivística reviste, bajo nuestra perspectiva, un doble criterio metodológico, y ambos igualmente necesarios (3). Por un lado, el análisis serio y riguroso de aquellas fuentes que directamente atañen al estudio positivista de los archivos y su disciplina. Y, por otro, el estudio de aquellas otras fuentes, que podríamos calificar como indirectas, de carácter histórico, literario y simbólico, y que son de utilidad para especificar la visión que de los archivos y de la archivística se tenía en un determinado momento histórico. Así pues - y sin especificar en este punto, dado que nos obligaría a extendernos demasiado - , para el Antiguo Régimen español, el estudio de la historia de la archivística y de los archivos estaría fundamentado en dos grandes vertientes.

Por un lado, la propiamente archivística. Esto es, el estudio de aquellas múltiples fuentes que analizan las diferentes prácticas de gobierno, de sus consecuentes prácticas documentales y de los diferentes archivos que irán surgiendo como consecuencia de aquellas. Pensemos, por tanto, en primer lugar, en fuentes de carácter específicamente archivístico, tales como los variados y heterogéneos documentos sobre la organización y arreglo de los documentos, lo que ha dado lugar a toda una literatura arbitrista de carácter archivístico; o los diferentes instrumentos de control e información que se formalizan en los archivos durante el Antiguo Régimen. En segundo lugar, las fuentes que explican el necesario análisis histórico-institucional, y que resultan ser de absoluta necesidad para la comprensión de la práctica documental y procedimental de las instituciones generadoras de archivos. En tercer lugar, las regulaciones jurídicas, esto es, las ordenanzas y normas reglamentarias, de provecho para el estudio del acceso, organización y custodia de los documentos. En fuentes de carácter teórico y conceptual, de las que se infieren resultados interesantes para discernir, por ejemplo, el buen hacer de los secretarios, escribanos o cronistas. Pensemos, en consecuencia, en autores tan divulgados como Álamos de Barrientos o Saavedra Fajardo. E, incluso - tal 
y como postulan los historiadores de la cultura escrita -, los inventarios de bibliotecas, dadas las vinculaciones entre el universo bibliotecario y archivístico, lo cual ocasiona que junto al material librario encontremos abundantes papeles - personales o familiares - y documentación de carácter archivístico.

Pero, al margen de estas fuentes, encontramos otras que, a pesar de su vinculación indirecta, nos resultan extremadamente útiles para la comprensión de la visión de los archivos y la archivística durante el Antiguo Régimen, que son de carácter histórico, literario o simbólico. Nos referimos, por un lado, a las fuentes tradicionales o narrativas, que incluyen: crónicas oficiales y no oficiales; memorias, diarios, epistolarios y correspondencia diplomática; papeles propagandísticos; gacetas, avisos y noticias; o relatos y memorias de viajeros. Y, por otro, a obras de carácter jurídico (como las actas de Cortes o los procesos judiciales), literario (obras literarias y piezas liminares) o simbólico-plástico (pensemos en la literatura emblemática).

Dichas fuentes son especialmente convenientes para el análisis de la visión - o percepción social- que de los archivos y la archivística se tenía en el Antiguo Régimen, dando respuesta a múltiples interrogantes sobre su función, su vinculación con la acción política, el uso cotidiano de los documentos, su utilización como garantía de la memoria escrita o su articulación con el proceso de escrituración. Se trata, pues, de analizar la representación imaginaria que se ha tenido de nuestra disciplina, porque en historia, lo imaginario es tan historia como la Historia con mayúsculas.

En definitiva, mediante el empleo de esta duplicidad de las fuentes conseguiremos dos propósitos: por un lado confeccionar un análisis pluridimensional de la historia de los archivos durante el Antiguo Régimen, configurando así una estructura histórica de la archivística de carácter global (dotada de una interna articulación que sistematice la compleja red de relaciones que nuestra disciplina tiene); y, por otro, consideramos que mediante el estudio de dichas fuentes desvelaremos aquellos factores de carácter sociocultural que han incidido en el desarrollo de la historia de la archivística, insertando nuestra disciplina en el contexto global histórico y administrativo.

Pues bien, una de las fuentes indirectas que consideramos imprescindible analizar, con objeto de establecer la percepción social que de los archivos y su contexto se tenía, son las memorias y relatos de viajeros.

\section{Memorias y relatos de viajeros}

Pero ¿qué son las memorias y relatos de viajeros? Para su análisis condensado intentaremos dar algunas indicaciones acerca de sus características peculiares como fuente, indagando dónde radica su interés histórico y dónde su problemática 
y dificultades de uso; cuáles son sus elementos básicos y constitutivos; y cuáles los recursos de que se vale para sus múltiples formas y finalidades.

La historiografía acerca de los viajes y viajeros durante la Edad Moderna es extraordinariamente amplia, fundamentalmente en las últimas dos décadas. Obviamente no realizaremos ahora ni siquiera un breve recordatorio de nombres, títulos y líneas de investigación. R. Foulché-Delbosch, Morel-Fatio, Farinelli, A. Bertrand, García Mercadal, Díez Borque, García Romeral... son solo algunos de los nombres que pueden citarse (4). Ciertamente la descripción de lugares y pueblos ha originado, desde la antigüedad, una multitud de géneros que tienen en común el "dar cuentas a los demás de la aventura personal" (5): Así, si rastreamos en nuestra literatura -desde los primeros poemas épicos hasta el Quijote-, observaremos cómo la significación que presenta la noción de "aventura" se encuentra comúnmente vinculada a la percepción conceptual que se detenta del concepto de viaje. Por otro lado, ya desde la Edad Media encontramos una literatura específica de viajes (6) al margen de algunas obras históricas que siguen como precepto estructural el viaje y la aventura (7). Al mismo tiempo, no hemos de olvidar que del siglo XVI datan las primeras guías de caminos en España, que permitían, aunque no sin dificultades, preparar cualquier desplazamiento (8).

Sin embargo - tal y como lo especifican en abundantes ocasiones los viajeros consultados - , viajar en el Antiguo Régimen no era fácil. Los problemas, en los que no nos detendremos, eran muchos (9). En primer lugar, la mala conservación de los caminos, ocasionada porque ninguna hacienda municipal podía soportar los costos de mantenimiento. Incluso, como a veces aseveran nuestros viajeros, en determinados lugares ni siquiera había caminos, o a lo sumo uno. De ahí que las perspectivas paisajísticas se repitan tantas veces en distintos viajeros. En segundo lugar, la inseguridad e indigencia en los viajes, donde el bandolerismo, el robo, la suciedad en las posadas o la falta de comida eran problemas de indudable importancia (Norberto Caimo, 1755):

Habiéndome puesto de nuevo en camino recorrí esa mañana una ruta encantadora... caminé seis horas para alcanzar un pueblo pequeño, llamado Blanco, donde he hecho una comida tan escasa, que ha servido más, según me parece, para irritar la gran necesidad que tenía que para disminuirla... Llegados por la noche a un pequeño lugar, no encontramos allí donde alojarnos... lo que nos obligó a continuar nuestra marcha durante dos horas, no viendo casi por dónde ir, por senderos pedregosos y bastante difíciles. Por fin [...] alcanzamos una posada, o por mejor decir una cabaña miserable, verdadero refugio de búhos y murciélagos. Un hombre gordo se levantó en camisa para recibirnos, llevando en la mano un farol cuya luz estaba dispuesta a apagarse. Con su recia y torpe voz, así como sus maneras aún más desagradables, más bien se le habría tomado por un animal que por un hombre. Fue preciso agarrarme a él para subir por una escalera medio rota a un cuarto sucio y más infecto que una cuadra. Había allí, en un rincón una cama muy estrecha, muy corta y muy sucia... No creo 
haber pasado en mi vida una noche peor. Además de los millares de insectos de toda especie que me destrozaban por todas partes, tuve toda la noche carreras y peleas de ratas, que no acabaron hasta el amanecer .

Por último, hay que tener en cuenta los imponderables geográficos y climáticos. España era un país muy difícil de atravesar (Antonio de Brunel, 1655):

La naturaleza parece haber puesto como una separación fija e indominable entre Vizcaya y Castilla la Vieja. Por eso ha sido necesario abrir el paso a fuerza de martillos, de cinceles y de minas [...].

Dicho esto, varias son las dificultades que encontramos para la utilización de las memorias y relatos de viajeros como fuente para la historia - también para la historia de la archivística: en primer lugar, su extraordinaria exhaustividad y diversidad. Exhaustividad, por el enorme número de textos que hay; y diversidad, porque según qué textos cambian los propósitos, el nivel intelectual de los autores, el público al cual se dirigen o las estrategias de su redacción. Ello ocasiona - y más para los siglos XVII y XVIII - la imposibilidad de ofrecer clasificaciones tipológicas, $\mathrm{o}$, si se quiere, la posibilidad de sugerir diferentes clasificaciones según el criterio que escojamos, y de las que aquí no haremos mención; en segundo lugar, y fruto de lo anterior por el carácter subjetivo de la fuente, cada viaje tiene unos objetivos y misiones peculiares. Así, son diferentes el relato de carácter comercial de Madame D’Aulnoy, la actividad práctica - a modo de guía - de Juan Bautista Labaña, el peculiar interés narrativo de Brunel o el tradicionalista y costumbrista de Bertua (10). Quizás, cabe destacar la misión diplomática. En este sentido, encontramos interesantes testimonios sobre instituciones del Estado, su organización y funcionamiento, las líneas políticas que se siguen, etc. En consecuencia, entendemos que los relatos de viajeros no deben utilizarse como única fuente para el conocimiento de las muchas facetas de una sociedad. Y una tercera consideración. Otra de las dificultades que constato para la utilización como fuente histórica de las memorias y relatos de viajeros es la extremada importancia que adquiere el relato (11). No en balde, al decir de algunos, se trata de un género literario específico. Y tanto es así que en ocasiones el investigador ha de realizar un esfuerzo para esclarecer qué parte del texto garantiza honradamente lo observado, y qué otras son simuladas y artificiosas. De manera que no en pocas ocasiones unos libros repiten lo que ya se dice en otros, deviniendo la labor del historiador en discernir qué obras de referencia se utilizan y por qué. Así, por ejemplo, Esteban de Silhouette presenta en el texto, por ejemplo, importantes indicios de lectura efectiva de viajeros, cronistas, teóricos, obras de referencia, tratados de historia. En muchas ocasiones cita su nombre.

El padre Labat, en obra titulada Viaje en España y en Italia no habla más que de Cádiz, Gibraltar y Sevilla. Este padre es un burlón por sus bromas no siempre son afortunadas, ni felices ni justas. Otras tres relaciones, la una de un viaje hecho en 1655; la otra, 
de un viaje hecho en 1659 por un consejero del Parlamento, y la tercera, impresa en 1717 con muy mediocres [...].

Llegados a este punto, varios podríamos decir que son los elementos constitutivos de las memorias de viajeros (Crivăt, 2003). En primer lugar, el itinerario se convierte en el elemento básico de la construcción de los relatos de viajes. En consecuencia, el texto llega a sistematizarse como un verdadero diario en el cual las llamadas espaciales y temporales devienen en constituir la unidad del texto. Ejemplo claro es el Itinerario del reino de Aragón de Juan Bautista Labaña en 1610-1611:

Miércoles, 27 de octubre. De Guadalajara a Taracena, una legua, y queda Iriépal un poco desviado mano derecha. De Tarazona a Valdenoches, dos leguas y de allí a Torija, una legua y media. El camino es por un valle estrecho, entre montes, y un espacio de huertas muy frescas...

En segundo lugar, la descripción. Esta se constituye como el elemento fundamental y organizador del itinerario. Ciudades, edificios y monumentos, obras de arte, costumbres, paisajes... En palabras de Anca Crivăt, "lo narrativo se disuelve en lo descriptivo, en cuya reconstrucción presentan los libros de viajes su valor historiográfico". Véase si no el siguiente ejemplo que describe la Biblioteca de El Escorial (Norbert Caimo, 1755):

Esta es verdaderamente incomparable por todo aquello que contiene de raro y de precioso. Pero tiene un defecto muy sorprendente en la puerta, que parece entera desde el exterior y de la que no se abre más de la mitad, porque por dentro está demasiado cerca del ángulo; eso quita la facilidad de una entrada conveniente. Todo el suelo es de mármol pulido, de dos colores, distribuido en compartimentos que produce un bellísimo efecto, y todo el contorno es de jaspe hasta la altura de ocho pulgadas [...].

Al mismo tiempo, los viajeros utilizan para conseguir sus propósitos determinados recursos: por un lado, el detalle de lo raro y exótico deviene en ser uno de los objetivos principales de nuestros textos. Se busca, pues, la sorpresa como efecto literario (Francisco Bertaut, 1659):

Me costaba trabajo creer lo que un librero, en cuya casa compré libros me dijo, que había dado la comedia a mariscal de Bairon, en versos burlescos a un fraile que la debía representar en su convento y que su mujer había prestado su vestido a uno de ellos para eso.

Por otro lado, el humor. La descripción de "lo otro", el uso en el lenguaje de la exageración y la hipérbole y el deseo de los viajeros por aquellas realidades distintas a las suyas y que les son novedosas son comunes en nuestros textos, como ocurre en este extracto de la Carta del mariscal de Bassompierre al señor de Puizieux a la muerte de 1621:

Señor. He hecho tan bien el cumplimiento del pésame que el rey me había ordenado, que salvo no haber llorado, todas las apariencias del desagrado y de la tristeza han 
aparecido sobre mi rostro, que abandona ahora esa falsa máscara, puesto que nada puede ya retardar mi regreso a Francia, adonde me voy con mil alegrías y mil deseos de servir bien a mi señor.

o en este otro de Norbert Caimo (1755, Universidad de Sigüenza)

He asistido a una tesis pública de medicina y anatomía. La principal cuestión que allí fue discutida fue saber de qué utilidad o de qué perjuicio sería al hombre tener un dedo más o un dedo menos. Esperaba que discutirían también si para gozar de una buena salud era preciso, al cortarse las uñas, comenzar por la mano derecha o por la izquierda, por el pulgar o por el meñique.

La utilización de prólogos es otro de los recursos utilizados. Se trata de un instrumento útil para captar a lector intentando conseguir así una cierta moralidad y reforzar la credibilidad de los relatos posteriores (Crivăt, 2003) (Enrique Cock, 1585):

Muchas causas me han movido, serenísimo Príncipe Felipe, para dirigirle estos Anales [...]. La primera, por haberme comenzado en el felicísimo principio de vuestra alteza [...]. La segunda es que por esto espero le serán muy agradables que a todos los hombres, y mayormente a los príncipes, suele ser gran consuelo acordarse de los trabajos pasados en la mocedad [...]. La tercera causa es que, habiendo yo trabajado casi diez años en las antigüedades de España para escribirlas con mucha verdad y diligencia $[\ldots]$.

En definitiva, los relatos de viajeros presentan abundantes datos sobre múltiples cuestiones, ofreciéndose, al mismo tiempo, interesantes valoraciones sobre diferentes cuestiones que nos permiten vislumbrar la historia imaginaria.

\section{La visión de los archivos en las memorias y relatos de viajeros}

Analizada la fuente, veamos a continuación cuál era la percepción que los viajeros tenían de los archivos y su contexto. Se trata, pues, como ya dijimos, de indagar acerca de la representación imaginaria que se ha tenido de nuestro objeto de estudio. Y en esta ocasión, como digo, a través de los viajeros. Para esta parte de la exposición, intentaremos explicar la articulación del trabajo realizado. Para ello, explicaremos sucintamente la metodología que practicamos y sistematizaremos los resultados, obviando el extenso caudal de datos que se extrajo.

\subsection{Criterios metodológicos}

Varios han sido, pues, los criterios metodológicos por los que optamos. En primer lugar, la Monarquía Hispánica del Antiguo Régimen fue lugar — tal y como ha estudiado Díaz Borque - de numerosos viajeros, aventureros, exploradores y curiosos, de dentro y fuera de la península. Justamente, el hecho de ser lugar imperial influye positivamente en ello. Sin embargo, para nuestro trabajo propusimos ajustarnos a los viajeros extranjeros, dado que son estos los que entran en contacto con realidades distintas de las que forman su habitual ambien-

Scire. $11: 1$ (en.-jun. 2005) 47-64. ISSN 1135-3761. 
te, y las descripciones suelen ser más específicas y sugerentes. Así, se han rastreado 52 memorias y relatos de viajeros que transcurren desde 1525 hasta 1755 , utilizando para ello la recopilación de García Mercadal (1999). Hemos intentado que la selección haya sido variada y, en consecuencia, los viajeros proceden de diversos países europeos -e incluso algún africano - con diferentes propósitos - comerciales, diplomáticos, políticos, novelescos, etc. - , con niveles intelectuales variados y con públicos distintos.

Por otro lado, hemos intentado recoger todo aquello que presenta alguna vinculación con el contexto archivístico. Así, se han escogido todos los datos que aparecen sobre archivos, ya sean estos de públicos, eclesiásticos, nobiliarios o personales. Pero también, todas aquellas cuestiones que aparecen vinculadas a la práctica documental. Nos referimos, fundamentalmente, a análisis históricos que explican los procedimientos administrativos de las diversas instituciones. Igualmente, se ha extraído lo concerniente a la cultura escrita, como factor sociocultural de la archivística. Lo escrito representa la necesidad del archivo, su función coercitiva y su importante cometido en las decisiones políticas.

El resultado de todo ello han sido más de cuatrocientas referencias, que intentaremos sistematizar a continuación muy resumidamente.

\subsection{Características}

Así pues, alejándonos de la confección de un estudio positivista en favor de un ejercicio razonado y deductivo, podríamos esquematizar en cinco las características que del contexto archivístico se extraen de las memorias y relatos de viajeros.

La primera característica tiene que ver con la instrumentalización política del archivo y el importante papel que representa la burocracia. Nos referimos fundamentalmente a los siguientes elementos. En primer lugar, la constatación de que el desarrollo de los archivos está ligado a la complejidad de las diferentes formas políticas. Dicha complejidad originará una mayor producción documental y una mayor presencia de personal técnico profesionalizado con responsabilidades gubernativas y judiciales. La burocracia, por consiguiente, favorecerá el surgimiento de procedimientos administrativos regularizados, convirtiéndose así en requisito preferente para convertir al archivo en un instrumento de privilegio y eficacia. Son numerosas las descripciones que ofrecen nuestros viajeros de las múltiples instituciones, pudiéndose entresacar datos de enorme valía sobre los procedimientos que se seguían en la comunicación administrativa: consejos, instituciones de justicia, diputaciones, cámaras de alcaldes, etc. Son descritos con todo lujo de detalles, ofreciéndonos múltiples datos de enorme valía para la práctica documental (Madame D’Aulnoy, 1680):

Scire. $11: 1$ (en.-jun. 2005) 47-64. ISSN 1135-3761. 
Todo el papel de oficio está sellado y cuesta más que el corriente. Hay una cierta época en la que se hace el reparto de los pleitos; los instruyen en Madrid, y pocas veces se sentencian; meten todos los documentos de una parte en un saco, los de la otra en otro, la instrucción en un tercero, y cuando es llegado el tiempo de distribuir los pleitos, los envían a los tribunales lejanos; de manera que muy a menudo son juzgados sin saber nada de ello; lo escriben en un registro donde el pleito fue enviado, y allí lo guardan con mucho secreto [...].

Asimismo, es constante la presencia de letrados, secretarios, burócratas, escribanos, alguaciles, notarios... que nos exhiben su trabajo (Antonio de Brunel, 1665):

Cuando Don Luis de Haro da audiencia a alguien, es recibido también por su Secretario Cristóbal que debe servir de intérprete. Se pone de rodillas y escribe [...].

Como resultado de todo ello, el archivo ha ocupado históricamente un puesto de poder. Consideramos que con tres características, al menos: favoreciendo el rigor y la agilidad administrativas; permitiendo un control de las actividades gubernativas o judiciales; y facultando un control sobre el conjunto de los particulares. En este sentido, y en palabras de Rodríguez de Diego, nuestros viajeros palpan "la función coercitiva del archivo", que está basada en tres elementos: su carácter patrimonial, (esto es, la consideración del archivo como atributo del rey) - "Simancas es una fortaleza que hay que ver desde Irún hasta Madrid. Es el Archivo del Rey [...]" (Camilo Borghese, 1594) —; su secretismo (es decir, el temperamento sacro del archivo) (Enrique Cock, 1585):

Simancas, Septimanca antiguamente llamada, es villa de hasta 500 vecinos, que tiene su asiento sobre la ribera occidental del río Pisuerga [...]. En lo más alto de la villa tiene su majestad una buena fortaleza, en cuyos aposentos bajos y debajo de la tierra están los archivos del Reino, donde se guardan con gran cuidado y secreto todas las escrituras públicas.

y su inaccesibilidad (Norberto Caimo, 1755):

He dejado a mi izquierda, a poca distancia, la villa, o mejor, la fortaleza de Simancas, construida sobre una colina muy elevada. Está rodeada de torres y de muros de tan gran fuerza que parece intomable. Allí es donde está el depósito de Archivos Reales de toda España.

Son interesantes igualmente las descripciones que ofrecen los viajeros sobre el rey y su forma de despachar. Veamos el siguiente ejemplo (Leonardo Donato, 1573):

Algunas propiedades particulares del Rey de España.

El rey negocia con gusto por escrito, y lo hace porque no gusta de tratar con muchos porque es más rápido en el escribir que cualquier secretario.

Ve todas las cosas suyas y todo lo sabe. [...] Lee con una candela cerca del lecho algunas horas antes de dormirse. Decía el nuncio del pontífice haber estado cinco 
años en la corte y no haber nunca hablado al rey en otra parte que en su escritorio, ni nunca de otro que de negocios, y que siempre la respuesta era dada de palabras generales. [...] El rey casi nunca habla con los suyos de la cámara. El nuncio decía que, finalmente, necesitaba negociar con el rey por billetes, esto es, por escrito; tan grande es su retiro.

O el que sigue (Tomás Contarini, 1593):

Usa [el rey] de extrema diligencia en el gobierno de sus estados y, quiere que todos los asuntos de alguna consecuencia pasen por sus manos. Todas las deliberaciones importantes le son enviadas por los consejeros, escritas sobre una hoja de papel con la mitad de margen, y en este pone su opinión, añadiendo, tachando y corrigiendo todo según su voluntad. Y cuando le queda tiempo, lo emplea en volver a ver y apostillar las súplicas y las demandas de los súbditos, y en otras escrituras de menor importancia; es lo que le ocurre algunas veces hacer durante tres y cuatro horas. Jamás descuida ninguna de esas ocupaciones, incluso cuando en el Escorial, y durante el viaje, trabaja con sus ministros, y revisa con cuidado los papeles que le son sometidos.

La segunda característica que deducimos es la visión del archivo como salvaguarda y garantía jurídica, esto es, la protección de los derechos de particulares e instituciones. Es muy frecuente, entre nuestros viajeros, el hecho de hacer constar la condición del documento escrito como el principal instrumento probatorio de cualquier derecho. Así, por ejemplo, para el hecho de viajar, son muy abundantes las referencias a contratos, pasaportes, etc. (Señor de la Melonnière, 1720):

Llegué por fin, después de muchas fatigas, al pueblo de San Román, al pie de la Estrella. Tenía, además de mi pasaporte, cartas del ministro para el juez del lugar. Ese buen hombre, no pudiendo concebir que gentes razonables quisieran ir a visitar el lago que está encima de la estrella, persuadidos de que los diablos han establecido allí su domicilio, me demostró algún recelo sobre la validez de mi pasaporte. Buscó varias cartas del Secretario de Estado para examinar la firma y, convino, al fin, en que la que yo llevaba era la verdadera.

Por otro lado, hay múltiples alusiones al notariado como personal que acredita la autenticidad del proceso de escriturado de un documento y se responsabiliza de su correcta custodia (Juan Bautista Labaña, 1610):

[...] subió al monasterio e hizo grandes exámenes con los pedazos del jarro, limpiándolos mucho y encerrándolos en la iglesia, guardando él las llaves y haciendo todas las diligencias exquisitas para seguridad de que ninguno llegase a los pedazos, los cuales, visitando después, halló que manaba de ellos el santo óleo como hasta allí había hecho, estando el jarro entero, y así acabó de creer en el milagro y estimarlo como tal, de lo que hizo un acta por un notario.

E igualmente, en nuestros textos encontramos copiosas menciones a registros varios, libros de ordenaciones, privilegios conservados. Todo ello, con objeto de favorecer la eficacia administrativa y la garantía jurídica de los derechos (Madame D’Aulnoy, 1680): 
Además de esta cámara, que está en Madrid, hay una en Sevilla, llamada la Casa de Contratación. Está compuesta por un Presidente y varios Consejeros de toga y espada, con los demás oficiales necesarios. Los Consejeros de espada conocen de las cosas que conciernen a la flota y a los galeones. Los otros Consejeros son los encargados de administrar justicia. Las apelaciones de ese tribunal van al Consejo de las Indias, en Madrid. En la Casa de Contratación de Sevilla se llevan registros donde se inscriben todas las mercancías que se envían a las Indias, y todas aquellas que de allí se traen, para impedir que el rey sea defraudado en sus derechos; [...].

La tercera característica que vislumbramos es la vinculación de los archivos con la memoria histórica. Nos referimos fundamentalmente a dos cuestiones: por un lado, constatamos la visión del documento de archivo como instrumento verificador del discurso histórico. De ahí la necesidad de que los documentos estén “arreglados" y se custodien adecuadamente (Enrique Cock, 1585):

Hay una escritura auténtica del libro del Rey Ramiro, escrita de su mano, que se guarda en el Archivo de Jaca, junto con otras, que demuestran lo dicho. Y otras a su lado dicen que el dicho Ramiro no fue más que elegido obispo y no consagrado.

Y una segunda cuestión que nos concierne. La historia es considerada como un instrumento de carácter político. En este sentido, son claros los intereses políticos de las monarquías europeas cada vez más preocupadas por cuestiones relacionadas con lo que hoy llamaríamos opinión pública e identidad nacional (Norberto Caimo, 1755):

Después de haber cenado de lo que había llevado conmigo, porque el posadero no tenía nada que darme, leí algunos papeles prestados. Ciertamente quiere hacer de esta tierra su rey, algo más de lo que realmente es. Quiere hacerla rica y poderosa cuando es pobre y apasionada.

Otra de las características que observamos en los relatos de viajes es lo que podríamos denominar práctica eclesiástica. Dejando para otra ocasión explicaciones más ponderadas, veámoslo ahora de forma sucinta: es sabido, por un lado, cómo los poderes religiosos han tenido un denodado empeño por transmitir la doctrina de modo unilateral y completo. Así, vemos, en ocasiones, cómo las pretensiones de conservación e intemporalidad del documento eclesiástico refuerzan su archivación y custodia (Gaspar Barreiros, 1547).

De los Milagros de Nuestra Señora tiene hecho por aquellos que devotamente a ella se encomendaron, un registro en la casa en la que están escritas muchos acontecimientos religiosos. Deben estos recordarse cómo fueron y conservarse en su tradición auténtica.

Asimismo, la administración eclesiástica con su significativo grado de cohesión y su complejidad administrativa servirán de modelo a la Corte, cuando esta comience a desarrollar articulaciones jurídico-administrativas complejas (Juan de Vandenesse, 1556): 
¿Por qué los eclesiásticos tienen aquí tanto poder? Su administración, tan bien proveída, debería ser ejemplo.

Por último, nuestros relatores no olvidan las importantes medidas organizativas en los archivos eclesiásticos desde el Concilio de Trento (duque de San Simón, 1721).

Entramos en la iglesia de la villa. Es amplia, alta y limpia. Pasamos después a la sacristía que se asoma tras una puerta al fondo. Vimos las reliquias y tesoros [...]. Los libros, tal y como es mandado, eran custodiados.

Y una última característica, de considerable trascendencia en nuestros viajeros. Aquella que tiene que ver con la cultura escrita. Es sabido cómo la escritura supuso la entrada en escena de nuevos agentes culturales y una nueva forma de transmisión de la información, sustituyendo a la memoria y a la palabra como elemento transmisor y verificador de los contenidos culturales. Son muchos y variados los aspectos que podríamos tocar dada la, cada vez mayor, conexión entre los historiadores de los archivos y los de la cultura escrita. Por ejemplo, la presencia de documentos de naturaleza privada de características misceláneas, lo cual demuestra una cierta cultura archivística (Madame D’Aulnoy, 1680):

He visto algunos papeles de Santa Teresa, escritos de su propia mano; su letra es legible, grande y mediocremente bella [...].

El Marqués de Liche tenía una biblioteca extremadamente curiosa, llena de los más hermosos manuscritos del mundo, conteniendo los despachos y los asuntos más importantes de toda la Monarquía desde Carlos V hasta el presente.

Asimismo, lo escrito adquiere una importancia inusitada, lo cual deviene en la formalización de una cierta mentalidad cultural - que en otro momento especificaremos como es debido - provocándose así una necesidad archivística (duque de San Simón, 1721):

En lo más alto del torreón estaba este famoso franciscano recluido [...]. Hice lo que pude por verle por la ventana, pero no pude conseguirlo. Había allí, por lo menos, una vista hermosa y le daban los libros que pedía, tanto de vino y carne como quería; pero no le dejaban ver a nadie ni nada con que pudiera ayudarse a escribir.

Igualmente, son continuas las referencias a tipos documentales, despachos, correos, postas, papel sellado, firma de papeles y custodia de los mismos, etc. Una prueba de la importancia de lo escrito puede ofrecérnosla el siguiente relato del día de la muerte de Felipe III y que narra el mariscal de Bassompietg en 1621:

El rey Felipe III falleció en el Palacio real de Madrid el miércoles, último día de marzo de 1621, a eso de las nueve de la mañana. Inmediatamente después el duque de Uceda, que era su Sumillers de Corps, fue a la habitación del Príncipe, puso una rodilla en tierra y le besó la mano, como a su Rey, y a continuación todos los grandes y principales señores que se encontraban allí presentes; después el duque presentó al Rey la llave de los gabinetes y escritorios del difunto Rey, su padre, y al mismo tiempo 
la arquilla y los sacos de sus papeles, el cual recomendó los pusiese entre las manos de don Baltasar de Zúñiga, que fue el primer acto del favor del dicho Don Baltasar de Zúñiga, y la ruina del duque de Uceda, el cual quiso al mismo tiempo salir del palacio, donde estaba alojado.

\section{Conclusión}

Permítasenos acabar muy brevemente con tres conclusiones. En primer lugar, la necesidad de emplear diversas fuentes para confeccionar el análisis pluridimensional de la historia de los archivos y su contexto durante el Antiguo Régimen. Las fuentes de carácter específicamente archivístico, aquellas otras que inciden en la vertiente histórico-institucional, las regulaciones jurídicas y otras fuentes de carácter teórico son imprescindibles. Pero también lo son otras de carácter histórico, literario y simbólico de especial conveniencia para el estudio de la representación imaginaria de nuestra disciplina. En segundo lugar, hemos constatado la importancia que tienen las memorias y relatos de viajeros para el estudio de la visión de los archivos en el Antiguo Régimen hispano. Nosotros diríamos que por dos razones: por un lado, por los datos positivos que se nos ofrecen sobre diversas cuestiones; $y$, por otro, por las valoraciones que se vislumbran del contexto archivístico. Y para terminar, una última conclusión. La instrumentalización política del archivo y el importante papel que representa la burocracia, la salvaguarda y garantía jurídica, la memoria histórica, la práctica eclesiástica y la cultura escrita son los factores socioculturales que, extraídos de forma deductiva de las memorias y relatos de viajeros, esquematizan el despliegue de la archivística de manera simultánea durante el Antiguo Régimen.

\section{Notas}

(1) Vid. al respecto Muñoz Gutiérrez (1994); Pederson (1994); Craig (1996); y Couture y Ducharme (1998-1999).

(2) Nosotros nos encargamos de sacar a la luz uno de ellos: Vivas Moreno (2001).

(3) Véase al respecto algunas reflexiones que realiza el sugerente estudio de Navarro Bonilla (2003). Al mismo tiempo, son adaptables las ideas que se vierten en los magníficos estudios siguientes: Bouza Álvarez (2001); Chartier (1992); Chartier (1995); y Chevalier (1976).

(4) La bibliografía sobre viajes y viajeros es amplísima. A modo de ejemplo podemos citar las siguientes por orden cronológico: Liske (1878); Foulché-Delbosc (1896); Morel Fatio (1878); Morel Fatio (1906); Guillon (1902); Bertrand (1920); Farinelli (1921); Claverie (1923); Martín Granizo (1927); Sánchez Rivero y Mariutti (1933); Sarrailh (1934); Álvarez Peña (1945); Swinburne (1946); García Mercadal (s. a.); Ribbans (1955); Fernández Álvarez (1956); Miquel (1967); y De la Puente (1968). A partir de los años setenta se produce un verdadero auge de la bibliografía que no podemos citar. Autores como Numa Broc, Ramón Boixareu, los espléndidos libros

Scire. 11 : 1 (en.-jun. 2005) 47-64. ISSN 1135-3761. 
de García Mercadal, Joan Salvador, Julio César Santoyo, Gaspar Gómez de la Serna, Oriol Valls, Joseph Massot, J. M Díez Borque, Ian Robertson, Marc-Aureli Vila, José Alderich, Joseph M. Marqués, María Enriqueta Soriano, Jordi Bolós i Masclans, Julio Caro Baroja, Patricia Show Fairman, Antonio Domínguez Ortiz, Carlos Martínez Shaw, Eva Serra, Roberto Fernández, Alejandro Sánchez, Montserrat Galera i Monegal, Joseph M. Farré i Pedrós, Antonio Pérez Gómez, Cristina Torres Suárez, Alfons Romero, Marta Garriga, Concha Casado, Antonio Carreira, Manuel Bernal Rodríguez, José Ramón Jiménez Corbatín, Lorenzo Díaz, Rafael d’Amat, Margarida Aritzeta, Jean-René Aymes, Julio Valdeón, etc. Una aproximación muy interesante a la historiografía: Moreno Chacón (1985). Asimismo, interesa citar los relevantes volúmenes de García Romeral.

(5) Expresión de Díez Borque (1975). Nos basamos en esta obra fundamentalmente para las primeras ideas que se vierten en este apartado.

(6) Por ejemplo, el Libro del conocimiento de todos los reinos e tierras, Embajada a Tamorlán de González de Clavijo, o Andanzas e viajes por diversas partes del mundo de Pero Tafur, traducciones de Marco Polo, etc.

(7) Véase, por ejemplo: La Fazienda de Ultramar o el Codex Calistinus para el siglo XII; de la época de Sancho IV, tenemos la Gran Conquista de Ultramar, y más tarde El Victorial o Crónica de Don Pero Niño, conde Buelna, etc.

(8) Viulluga, Juan de. Repertorio de todos los caminos de España, Medina del Campo, 1546; Meneses, Alonso de. Repertorio de caminos, 1576; u otras de Octavio Cotogno, Martín Zellerius, etc.)

(9) Véase una buena síntesis para esta temática en Alvar Ezquerra (1989). Nos basamos en este capítulo en parte.

(10) Véase al respecto las elucubraciones de Blasco Castineyra (1991). Igualmente, Blasco Castineyra (1991, pp. 168-170).

\section{Referencias}

Alvar Ezquerra, A. (1989). Viajes, posadas, caminos y viajeros. // Alcalá-Zamora, J. A. (dir.). La vida cotidiana en la España de Velázquez. Madrid: Temas de Hoy, 1989.

Álvarez Peña, C. (1945). Un viajero italiano del siglo XVI en Lérida. // Lleida. IV (1945) 45-73.

Bertrand, A. (1920). Voyageurs allemands en Espagne (fin du XVIIIe siècle et debut du XXe siècle. // Bulletin Hispanique. XXII (1920) 37-50.

Blasco Castineyra, S. (1991). La imagen literaria de El Escorial en el siglo XVIII. Reflexiones sobre las fuentes del viaje ilustrado. // Cuadernos de Historia Moderna de la Universidad Complutense de Madrid. 12 (1991) 167-182.

Bouza Álvarez, Fernando de (2001). Corre manuscrito: una historia cultural del Siglo de Oro. Madrid: Marcial Pons, 2001.

Chartier, Roger (1992). El mundo como representación. Historia cultural: entre práctica y representación. Barcelona: Gedisa, 1992. 
Chartier, Roger (1995). Sociedad y escritura en la Edad Moderna. México: Instituto Mora, 1995.

Chevalier, Máxime (1976). Lecturas y lectores en la España del siglo XVI y XVII. Madrid: Turner, 1976.

Claverie, Ch. (1923). Relation d'un voyage en Espagne (1612). // Revue Hispanique. LIX (décembre 1923) 359-555.

Couture, C.; Ducharme, D. (1998-1999). La recherche en archivistique: un état de la question. // Archives. 30:3-4 (1998-1999) 11-37.

Craig, B. L. (1996). Serving the truth: the importance of fostering archives research in education programmes, including a modest proposal for partnerships with the workplace. // Archivaria. 42 (1996) 105-117.

Crivăt, Anca (2003). Los libros de viajes de la Edad Media española. Bucarest: Universidad, 2003. URL: <http://www.unibuc.ro/eBooks/filologie/AncaCrivat/>. (Consultado: 12-2004.

De la Puente, J. (1968). La visión de la realidad española en los viajes de don Antonio Ponz. Madrid: Moneda y crédito, 1968.

Díez Borque, J. Ma (1975). El viaje en la literatura. // La sociedad española y los viajeros del siglo XVII. Madrid: Sociedad General Española de Librería, 1975.

Farinelli, A. (1921). Viajes por España y Portugal desde la Edad Media hasta el siglo XX. Divagaciones bibliográficas. Madrid: Centro de Estudios Históricos, 1921.

Fernández Álvarez, M. (1956). Aportaciones a la historia del turismo en España. Relatos de viaje desde el Renacimiento hasta el Romanticismo. Madrid: Ediciones de la Secretaría General del Ministerio de Información, 1956.

Foulché-Delbosc, R. (1896). Bibliographie des voyages en Espagne et Portugal. // Revue Hispanique. III (1896) 1-349.

García Mercadal, J. (1999). Viajes de extranjeros por España y Portugal. Valladolid: Junta de Castilla y León, Consejería de Educación y Cultura, 1999, 6 vols. Vols II, III y IV.

García Mercadal, J. (s. a.). España vista por los extranjeros. Relaciones de viajeros desde la edad más remota hasta el siglo XVI. Madrid: Biblioteca Nueva, s. a.

García Romeral, C. (s. a.). Bio-bibliografía de viajeros españoles. Madrid: Ollero y Ramos, s. a. Varios vols.

Gimeno Blay, Francisco (1999). De las ciencias auxiliares a la historia de la cultura Escrita. Valencia: Universidad de Valencia, 1999.

Guillon, E. (1902). Deux voyages en Espagne au XVIIe siècle. // Revue Hispanique. IX (1902) 509-513.

Liske, J. (1878). Viajes de extranjeros por España y Portugal en los siglos XV, XVI y XVII. Madrid: Casa Editorial de Medina, 1878 (traducido y anotado por don Félix Rozanski).

Maczak, A. (1996). Viajes y viajeros en la Europa moderna. Barcelona, 1996.

Martín Granizo, L. (1927). Aportaciones bibliográficas. Viajeros y viajes de españoles, portugueses e hispanoamericanos. Madrid: Revista de la Sociedad Geográfica, 1927.

Scire. $11: 1$ (en.-jun. 2005) 47-64. ISSN 1135-3761. 
Martínez Shaw, Carlos (1982). El llibre de viatges com a font històrica // L'avenç. 51 (1982) 46-48.

Miquel, F. A. (1967). Viatge a Catalunya d'un Conseller del rei de França l'any 1603. Barcelona: Dalmau, 1967.

Morel Fatio, A. (1878). L'Espagne au XVIe et XVII siècle: Documents historiques et littéraires. Heilbronn: Henninger Fréres, 1878.

Morel Fatio, A. (1906). Études sur l'Espagne. París: Honoré Champion, 2n sèrie, 1906.

Moreno Chacón, M. (1985). Visión historiográfica de los viajes por España en la Edad Moderna. // Revista d'Historia Moderna Manuscrits. 7 (1985) 189-212.

Muñoz Gutiérrez, C. (1994). The state of research in archival science. // Archivum 39 (1994) 530-532.

Navarro Bonilla, Diego (2003). La imagen del archivo: representaciones y funciones en España (siglos XVI y XVII). Gijón: Trea, 2003.

Pederson, A. E. (1994). Development of research programs. // Archivum. 39 (1994) 312359.

Ribbans, G. W. (1955). Catalunya i València vistes pels viatgers anglesos del segle XVIIIè. Barcelona: Barcino, 1955.

Sánchez Rivero, A.; Mariutti, A. (1933). Viaje de Cosme de Médicis por España y Portugal (1668-1669). Madrid: CEH, 1933.

Sarrailh, J. (1934). Voyageurs français au XVIIIe siècle. // Bulletin Hispanique. XXXVI (1934) 29-70.

Swinburne, H. (1946). Viaje por Cataluña en 1755. Barcelona: José Porter, 1946.

Vega Domínguez, J. (2001). Universidades, archivos y bibliotecas en las memorias y relatos de viajeros. // Vivas Moreno, A. Universidades y Archivos Universitarios. Badajoz: Universidad de Extremadura, 2001. 59-100.

Vivas Moreno, A. (2001). Lecturas y documentos históricos en Archivos: un informe del s. XIX para la organización del Archivo de la Universidad de Salamanca. Badajoz: Universidad de Extremadura, 2001. 